Aus dem Department of Physics der University of Michigan, Ann Arbor, Mich. und aus dem Institut für Theoretische Physik der Rheinisch-Westfälischen Technischen Hochschule Aachen

\title{
Kirchhoff-Youngsche Theorie der Beugung elektromagnetischer Wellen
}

\author{
IN MEMORIAM ARNOLD SOMMERFELD \\ anläßlich seines neunzigsten Geburtstages \\ Von \\ O. Laporte und J. Meixner \\ Mit 2 Figuren im Text \\ (Eingegangen am 6. August 1958)
}

Der Youngschen Deutung der Beugungserscheinungen liegt die Vorstellung zugrunde, daß das Licht am Rand der beugenden Öffnung ,eine Art von Reflexion" erleidet. Für skalare Wellen läßt sich diese Auffassung streng aus der Kirchhoffschen Näherungstheorie der Beugung begründen, und auch ,die Art der Reflexion“ lä.ßt sich genau definieren. - Der Gedankengang des Beweises kann auf den Fall elektromagnetischer Wellen übertragen werden und führt dort ebenfalls zu einer Darstellung des gebeugten Feldes durch das direkte, geometrisch-optisch berechnete Licht und einer zusätzlichen Strahlung, die als Integral über den Rand der Öffnung berechnet wird und als Reflexion am Rand im Sinne von Young interpretiert werden kann. - Die Ausrechnung der zweifachen Kirchhoffschen Beugungsintegrale über die Öffnung kann somit auch für elektromagnetische Felder auf die Berechnung einfacher Integrale längs des Öffnungsrandes zurückgeführt werden.

\section{Einleitung}

In der Theorie der Beugung spielt das Helmholtzsche Integral eine bedeutende Rolle. Es erlaubt das Wellenfeld in einem Halbraum $\mathrm{H}_{2}$ (dessen Begrenzung nicht notwendig eben zu sein braucht) zu berechnen, wenn die Werte und die Normalableitung des Wellenfeldes auf der Begrenzung des Halbraumes bekannt sind und wenn noch eine geeignete Bedingung im Unendlichen, etwa die Ausstrahlungsbedingung, vorgegeben ist.

Vom Helmholtzschen Integral wird Gebrauch gemacht, wenn man die Beugung einer im Halbraum $H_{1}$ einfallenden Welle an einem Schirm $\sigma$ berechnen will, welcher zusammen mit der Öffnung $\bar{\sigma}$ die Grenze zwischen den Halbräumen $H_{1}$ und $H_{2}$ bilden soll (s. Fig. 1). Man erhält eine Näherung des Wellenfeldes in $H_{2}$, wenn man auf der Schattenseite von $\sigma$ der Wellenfunktion und ihrer Normalableitung den Wert Null gibt, während sie in der Öffnung $\bar{\sigma}$ zusammen mit ihrer Normalableitung mit der 
ungestörten einfallenden Welle übereinstimmend angenommen wird. Diese Näherung wird als Kirchhoffsche Näherung der Beugungstheorie bezeichnet. Sie ist um so besser, je größer die Abmessungen der Öffnung und des Schirmes im Vergleich zur Wellenlänge sind. Sie versagt, wenn diese Abmessungen von der Größenordnung der Wellenlänge oder kleiner sind.

Näherungen dieser Art sind in der praktischen Optik sehr nützlich und werden auch im Mikrowellenbereich zunehmend wichtiger. Ihre

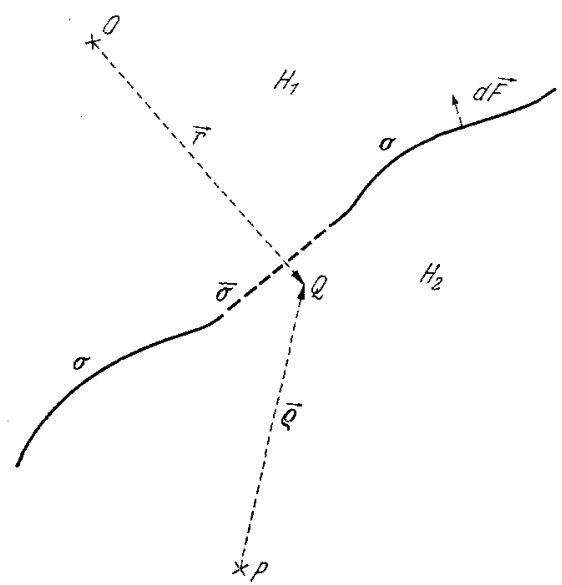

Fig. 1. Zur Beugung am Schirm $\sigma$ mit der Öffnung $\bar{\sigma}$. $O$ Lichtquelle, $P$ Beobachtungspunkt, $Q$ variabler Punkt. Die beiden Halbräume $H_{1}$ und $H_{2}$ werden durch die Fläche $\sigma+\bar{\sigma}$ getrennt praktische Auswertung wird indessen dadurch kompliziert, daß zweifache Integrale auszuführen sind. Wie MaGgI ${ }^{1}$ und RubINOwICZ ${ }^{2}$ gezeigt haben, kann man diese Integrale in einfache Integrale längs desÖffnungsrandes verwandeln. Besonders elegant und physikalisch einleuchtend ist eine neue Methode von Rubinowicz ${ }^{3}$, welche dieseTransformation durch Einführung eines neuen Vektorpotentials für gewisse bilineare Felder bewerkstelligt.

Die so erhaltenen einfachen Integrale lassen sich wegen der ohnehin in der Kirchhoffschen Näherung enthaltenen Annahme kleiner Wellenlängen häufig bequem nach der Sattelpunktsmethode auswerten, und man erhält so eine übersichtliche Darstellung des gebeugten Feldes bei kleinen Wellenlängen.

Dieser Übergang von der Kirchhoffschen Näherung zum Randintegral von RuBINowicz ist bisher nur für skalare Wellen durchgeführt. Ein entsprechender Übergang ist jedoch auch für die Theorie der Beugung elektromagnetischer Wellen möglich, soweit sie von einer Näherung ausgeht, die der Kirchhoffschen Näherung entspricht. Diese Näherung ergibt sich in analoger Weise aus den Larmor-Tedoneschen Formeln ${ }^{4,5}$.

Zweck der folgenden Ausführungen ist es, diesen Übergang durchzuführen.

\footnotetext{
I MAGgr, G.A.: Ann. di Mat. IIa 16, 21 (1888).

2 Rubinowicz, A.: Ann. d. Phys. 53, 257 (1917); 73, 399 (1924).

a Rubinowicz, A.: Acta phys. Polonica 12, 225 (1953).

4 Larmor, J.: Proc. Lond. Math. Soc. (2) 1, 1 (1903).

5 Tedone, O.: Atti Accad. Naz. Lincei, Rend., Ser. V 26, 286 (1917).
} 


\section{Maxwellsche Gleichungen. Dipolfeld und Bezeichnungsfragen}

Die Maxwellschen Gleichungen für harmonische Vorgänge mit der Zeitabhängigkeit $e^{-i \omega t}$ lauten in konventioneller Schreibweise

$$
\operatorname{rot} \mathbb{E}=i \omega \mu \mathfrak{S}, \quad \operatorname{rot} \mathfrak{S}=-i \omega \varepsilon \mathbb{E} .
$$

Wir wollen sie aber von jetzt ab mit Feldvektoren schreiben, die sich von den in (2.1) benutzten durch Faktoren $\sqrt{\varepsilon}$ und $\sqrt{\mu}$ unterscheiden. Die Komponenten von $\sqrt{\varepsilon}\left[\right.$ seien $E_{\alpha}$, jene von $\sqrt{\mu}$ sg seien $H_{\alpha}$ genannt. Unter Benutzung der Wellenzahl

kann man dann schreiben

$$
\sqrt{\varepsilon \mu} \omega=k
$$

$i k H_{j}=-\varepsilon_{j \alpha \beta} \frac{\partial E_{\alpha}}{\partial x_{\beta}} ; \quad i \hbar E_{j}=\varepsilon_{j \alpha \beta} \frac{\partial H_{\alpha}}{\partial x_{\beta}} ; \quad \frac{\partial E_{\alpha}}{\partial x_{\alpha}}=0 ; \quad \frac{\partial H_{\alpha}}{\partial x_{\alpha}}=0$.

Alle Indizes laufen von 1 bis 3 , über doppelt vorkommende Indizes ist zu summieren. $\varepsilon_{i j k}$ ist der dreifach antisymmetrische Einheitstensor, $\varepsilon_{123}=1$ etc. Sein Gebrauch ließe sich vermeiden, wenn man willens wäre, die magnetische Feldstärke als antisymmetrischen Tensor zweiten Ranges zu schreiben. Davon ist jedoch hier abgesehen.

Um das Dipolfeld zu erhalten, gehen wir von einem Hertzschen Vektor $\vec{Z}$ aus, von dem die Feldvektoren in der üblichen Weise abgeleitet werden:

$$
H_{j}=i k \varepsilon_{j \alpha \beta} \frac{\partial Z_{\alpha}}{\partial x_{\beta}}, \quad E_{j}=\frac{\partial^{2} Z_{\alpha}}{\partial x_{j} \partial x_{\alpha}}+k^{2} Z_{j},
$$

wobei alle Komponenten von $\vec{Z}$ die Wellengleichung

$$
\frac{\partial^{2} Z_{j}}{\partial x_{\alpha} \partial x_{\alpha}}+k^{2} Z_{j}=0
$$

erfüllen müssen ${ }^{\star}$. Der Hertzsche Vektor trägt jetzt aber natürlich Tensorcharakter $Z_{i j}$ und infolgedessen werden auch die Feldstärken Tensoren $H_{i j}$ und $E_{i j}$. Es handelt sich eben um die $i$-te Komponente des Feldes eines Dipoles, der entlang der $j$-ten Koordinatenachse orientiert ist. Wir setzen dementsprechend

wo

$$
\left.\begin{array}{l}
Z_{i j}=f(r) \delta_{i j}, \\
f(r)=\frac{e^{i k r}}{r},
\end{array}\right\}
$$

* Beim Einsetzen von (2.3) in (2.2) muB man benutzen, daß

$$
\varepsilon_{\alpha i j} \varepsilon_{\alpha m n}=\delta_{i m} \delta_{j n}-\delta_{i n} \delta_{j m}
$$

gilt. $\delta_{j n}$ ist das Kroneckersche Symbol. 
was jedenfalls (2.4) befriedigt. Hier ist $r$ der Abstand eines festen Punktes von einem beweglichen Punkte, dessen Koordinaten von einem beliebigen Ursprung aus $x_{i}$ sein mögen (s. Fig. 1). Bei Differentiation nach $x_{i}$ treten dann die Komponenten von $r$ entlang den $x_{i}$, die mit $r_{i}$ bezeichnet werden mögen, auf, so daß z.B.

$$
\frac{\partial f}{\partial x_{i}}=\frac{1}{r} \frac{\partial f}{\partial r} r=\AA_{i}
$$

Der kleine Kreis über $f$ bedeutet demnach den Operator $\frac{1}{r} \frac{d}{d r}$, dessen Einführung viele Vorteile mit sich bringt. Die gewöhnliche Differentialgleichung, der $f$ genügt, kann mittels dieses Operators so geschrieben werden:

$$
r^{2} \stackrel{\circ}{f}+3 \stackrel{\circ}{f}+k^{2} f=0 .
$$

Ein $f$ mit $n$ Kreisen ist natürlich proportional einer Zylinderfunktion mit halbzahligem Index.

Jetzt wird der Hertzsche Tensor (2.5) in die Ausdrücke (2.3) eingesetzt, und es ergibt sich ohne weiteres

$$
\left.\begin{array}{l}
H_{i p}=i k \varepsilon_{i p \alpha} \gamma_{\alpha} \stackrel{\circ}{f}(r), \\
E_{i p}=\stackrel{\circ}{\dagger} r_{i} \gamma_{p}+\left(\dot{f}+k^{2} f\right) \delta_{i p} .
\end{array}\right\}
$$

Beide Feldvektoren werden also Tensoren, der magnetische antisymmetrisch, der elektrische symmetrisch in den Indizes $i, p$. Die obigen Ausdrücke sind also die $i$-ten Komponenten des Feldes eines entlang der $p$-ten Koordinatenrichtung weisenden Dipols.

\section{Ableitung des Rubinowiczschen Vektorpotentials}

In diesem Abschnitt soll die schöne Rubinowiczsche Idee dargestellt werden, aber in einer Art, die die Verallgemeinerungsmöglichkeit von der skalaren auf die vektorielle Beugung hervorhebt. Für den skalaren Fall legen wir die Gleichungen erster Ordnung des Schalls zugrunde, die aus der Linearisierung der hydrodynamischen Gleichungen entstehen. Diese sind, wenn wir das Verhältnis des Dichtezuwachses zur Ruhedichte mit $s$, das Verhältnis der Gasgeschwindigkeit zur Schallgeschwindigkeit mit $\vec{u}$ bezeichnen:

$$
\left.\begin{array}{l}
\frac{\partial u_{v}}{\partial x_{v}}=i k s, \\
\frac{\partial s}{\partial x_{j}}=i k u_{j} .
\end{array}\right\}
$$

Durch Annahme der üblichen Zeitabhängigkeit $\exp (-i \omega t)$ sind die Zeitableitungen weggefallen. Nun muß ein adjungiertes Gleichungssystem postuliert werden, dessen abhängige Variable mit $\bar{s}$ und $\bar{u}_{i}$ 
bezeichnet sein mögen. Dieses kann auf zwei, sich durch ein Vorzeichen unterscheidende Arten geschehen, eine Tatsache, der wir durch Einführung einer Größe $\eta= \pm 1$ gerecht werden. Die adjungierten Gleichungen sind also

$$
\left.\begin{array}{l}
\frac{\partial \bar{u}_{\nu}}{\partial x_{y}}=i k \eta \bar{s}, \\
\frac{\partial \bar{s}}{\partial x_{i}}=i k \eta \bar{u}_{i} .
\end{array}\right\}
$$

Nach Multiplikation dieser vier Gleichungen bzw. mit $\bar{s},-\eta \bar{u}_{i},-\eta s, u_{i}$ und Summierung über $i$ ergibt sich:

$$
\frac{\partial}{\partial x_{\nu}}\left(\bar{s} u_{\nu}-\eta \bar{u}_{\nu} s\right)=0 .
$$

Der divergenzfreie Vektor ${ }^{\star}$

$$
V_{i}=i k\left(s u_{i}-\eta \bar{u}_{i} s\right)
$$

ist bilinear in den beiden willkürlichen Lösungen $s, \bar{u}_{i}$ und $\bar{s}, \bar{u}_{i}$ von (3.1) und (3.2). Er läßt sich auch in folgender Weise schreiben

$$
V_{i}=\bar{s} \frac{\partial s}{\partial x_{i}}-s \frac{\partial \bar{s}}{\partial x_{i}} .
$$

Wenn man für $s, \widetilde{s}, u_{i}, \bar{u}_{i}$ die Punktquellenlösungen einsetzt, d.h.

so wird

$$
\begin{aligned}
& s=\frac{e^{i k r}}{r}, u_{i}=\frac{1}{i k} \frac{\partial}{\partial x_{i}} \frac{e^{i k r}}{r}, \\
& \bar{s}=\frac{e^{i k e}}{\varrho}, \overline{u_{i}}=\frac{1}{i k \eta} \frac{\partial}{\partial x_{i}} \frac{e^{i k \varrho}}{\varrho},
\end{aligned}
$$

$$
V_{i}=\frac{e^{i i k} \varrho}{\varrho} \frac{\partial}{\partial x_{i}} \frac{e^{i k r}}{r}-\frac{e^{i k \pi}}{\gamma} \frac{\partial}{\partial x_{i}} \frac{e^{i k e}}{\varrho}
$$

oder in der Bezeichnung von (2.5)

$$
V_{i}=f(\varrho) \stackrel{\circ}{f}(r) r_{i}-\stackrel{\circ}{f}(\varrho) f(r) \varrho_{i} .
$$

Dieser Vektor noch mit einem Flächenelement der Beugungsöffnung multipliziert, tritt gerade unter dem Integral bei der Kirchoffschen Näherung auf. Wir erinnern noch daran, daB bei der Herleitung der Kirchhoffschen Integralformel von der Divergenzfreiheit eines Ausdrucks der Form (3.5) bzw. (3.4) Gebrauch gemacht wird, wobei $s$ die Lösung des Beugungsproblems, $\bar{s}$ eine Sondenfunktion der Gestalt (3.7) ist.

* Es gibt auch einen Tensor mit verschwindender Tensordivergenz, Dieser ist

$$
T_{i j}=\bar{u}_{i} u_{j}+\bar{u}_{j} u_{i}-\eta \delta_{i j} \bar{s} s-u_{i} \bar{u}_{j} \delta_{i j}
$$


$\mathrm{Zu}$ den geometrischen Verhältnissen sei nochmals betont: Man hat zwei vorläufig feste Punkte, der Ort des Pols $O$ und der Aufpunkt $P$. Zwischen beiden ist ein beweglicher Punkt, dessen Abstandsvektoren von den festen Punkten $r_{i}$ und $\varrho_{i}$ sind. Über ihn wird integriert, bzw. nach seinen Koordinaten wird differenziert.

Auf Grund von (3.3) kann man nun den Vektor (3.4) bzw. (3.8) aus einem Vektorpotential oder, wie man in der Tensorsprache sagt, von einem antisymmetrischen Tensor $A_{i j}=-A_{j i}$ ableiten:

$$
V_{i}=\frac{\partial A_{i \alpha}}{\partial x_{\alpha \alpha}} .
$$

Für $A_{i j}$ macht man den folgenden naheliegenden Ansatz:

$$
A_{i j}=\left(r_{i} \varrho_{j}-\varrho_{i} r_{j}\right) f(r) f(\varrho) F(r, \varrho) .
$$

Der erste Faktor ist ganz unumgänglich, denn kein anderer antisymmetrischer Ausdruck läßt sich aus den zur Verfügung stehenden Vektoren herstellen. Weiterhin, da z.B.

$$
f(r)=\left(i k-\frac{1}{r}\right) \frac{1}{r} f(r),
$$

kann man die Faktoren $f(r), f(\varrho)$ im Vektor $V_{i}$ nicht anders als im Ansatz (3.10) vorweggenommen, hervorbringen. Die eigentliche Unbekannte ist die in $\vec{r}$ und $\vec{\varrho}$ symmetrische Funktion $F(r, \varrho)$. Für sie macht nun RubiNowicz den wesentlich spezielleren Ansatz der Unabhängigkeit von $k$; d.h. die in (3.8) vorkommenden Glieder erster Ordnung in $k$ sind lediglich durch die Differentiation der Exponenten von $f(r)$ und $f(\varrho)$ geschaffen. Dadurch wird die Bestimmung der Funktion $F(r, \varrho)$ nicht durch eine Differentialgleichung, sondern algebraisch möglich. Das Resultat ist

mit der Abkürzung

$$
F(r, \varrho)=\frac{1}{\Lambda}
$$

$$
\Lambda=r \varrho+r_{\nu} \varrho_{v} .
$$

Die Größe $A$ ist der charakteristische Nenner des Rubinowiczschen Integrals.

Die Kirchhoffsche Näherung für eine einfallende Kugelwelle (Quellpunkt $O$, Fig. 1) ergibt sich in bekannter Weise zu

$$
4 \pi u(P)=\int V_{i} d F_{i},
$$

wobei rechts über die Öffnung integriert wird. Setzt man in diesem Integral für $V_{i}$ den Ausdruck (3.9) ein, so kann man den Stokesschen Satz anwenden und das Flächenintegral auf ein Linienintegral über den Rand 
der beugenden Öffnung zurückführen. Der Faktor $1 / A$ in $A_{i j}$ bringt jedoch eine folgenschwere Modifikation: Er ist singulär, wenn die Vektoren $\gamma_{j}$ und $\varrho_{j}$ entgegengesetzt gerichtet sind. Man muß daher vor Anwendung des Stokesschen Satzes den singulären Teil des Integranden abspalten, falls die Verbindungslinie $O P$ die Öffnung durchsetzt. Dies gibt einen zusätzlichen Beitrag neben dem Randintegral, der jedoch sehr einfach ist; er ist nämlich gleich dem direkten Licht von $O$ aus.

\section{Die divergenzfreien bilinearen Vektoren für die Maxwellschen Gleichungen}

Wie aus dem vorhergehenden Abschnitt hervorgeht, ist die Divergenzfreiheit des Vektors (3.8) entscheidende Voraussetzung sowohl für die Herleitung des Kirchhoffschen Integrals als auch für seine Umwandlung in ein Linienintegral nach Rubinowicz. Wenn man diese Ergebnisse auf den Fall elektromagnetischer Felder übertragen will, muß daher der erste Schritt darin bestehen, divergenzfreie Vektoren $\mathrm{zu}$ finden, die bilinear in den Größen $E_{\alpha}, H_{\alpha}$ einerseits, $\bar{E}_{\alpha}, \bar{H}_{\alpha}$ andererseits sind. Dabei ist $E_{\alpha}, H_{\alpha}$ eine beliebige Lösung der Maxwellschen Gln. (2.2), während $\bar{E}_{\alpha}, \bar{H}_{\alpha}$ eine beliebige Lösung der adjungierten Maxwellschen Gleichungen

$$
i k \bar{H}_{j}=\eta \varepsilon_{j \alpha \beta} \frac{\partial \bar{E}_{\alpha}}{\partial x_{\beta}}, \quad i k \bar{E}_{j}=-\eta \varepsilon_{j \alpha \beta} \frac{\partial \bar{H}_{\alpha}}{\partial x_{\beta}}
$$

darstellt. Wie in (3.2) kann $\eta$ irgendeinen der beiden Werte $\pm \mathbf{1}$ annehmen.

Durch Multiplikation der beiden GIn. (2.2) und der beiden (4.1) in geeigneter Weise erhält man zwei Vektoren, die divergenzfrei sind. Und zwar, wenn man der Reihe nach (2.2) und (4.1) mit $-\bar{E}_{j}, \eta \bar{H}_{j}$, $-\eta_{j} E_{i}, H_{j}$ multipliziert, erhält man $\operatorname{div} V^{(1)}=0$, und wenn man wiederum der Reihe nach mit $\eta \bar{H}_{j}, \bar{E}_{j},-\eta H_{j},-E_{j}$ multipliziert und über $i$ summiert, erhält man $\operatorname{div} V^{(2)}=0$.

Dabei sind $V^{(1)}$ und $V^{(2)}$ Vektoren mit den Komponenten

$$
\begin{aligned}
& V_{j}^{(1)}=\varepsilon_{j \alpha \beta}\left[E_{\alpha} \bar{E}_{\beta}+\eta H_{\alpha} \bar{H}_{\beta}\right], \\
& V_{j}^{(2)}=\varepsilon_{j \alpha \beta}\left[H_{\alpha} \bar{E}_{\beta}-\eta E_{\alpha} \bar{H}_{\beta}\right] .
\end{aligned}
$$

Diese sind übrigens schon bekannt und bilden die Grundlage der Reziprozitätstheoreme der elektromagnetischen Wellentheorie $\left(\text { LORENTZ }^{6}\right)^{\star}$.

* Entsprechend der Anmerkung S. 133 sei erwähnt, daß es noch zwei symmetrische Tensoren gibt, deren Tensordivergenzen auch verschwinden. Diese sind

$$
\begin{aligned}
& T_{i j}^{(1)}=E_{i} \bar{E}_{j}+E_{j} \bar{E}_{i}+\eta\left(H_{i} \bar{H}_{j}+H_{j} \bar{H}_{i}\right)-\delta_{i j}\left(E_{\alpha} \bar{E}_{\alpha}+\eta H_{\alpha} \bar{H}_{\alpha}\right), \\
& T_{i j}^{(2)}=E_{i} \bar{H}_{j}+E_{j} \bar{H}_{i}-\eta\left(H_{i} \bar{E}_{j}-H_{j} \overline{E_{i}}\right)+\delta_{i j}\left(\eta H_{\alpha} \bar{E}_{\alpha}-E_{\alpha} \bar{H}_{\alpha}\right) .
\end{aligned}
$$

${ }^{6}$ Lorentz, H.A.: Nederl. Akad. Wet., Verslag Afd. Natuurk. 4, 176 (1896). 
Das vektorielle Analogon der Kirchhoffschen Theorie resultiert nun, wenn man für die überstrichenen Feldvektoren die eines Dipols im $\mathrm{Ab}$ stand $\varrho_{i}$ und für die nicht-überstrichenen Feldvektoren die eines Dipols im Abstand $r_{i}$ einsetzt (s. Fig. 1). Die letzteren sind schon in (2.8) vermerkt, die anderen sind entsprechend:

$$
\left.\begin{array}{l}
\bar{H}_{i q}(\vec{\varrho})=-i k \eta \varepsilon_{i q \alpha} \varrho_{\alpha} \stackrel{\circ}{f}(\varrho), \\
\bar{E}_{i q}(\vec{\varrho})=\stackrel{\infty}{f}(\varrho) \varrho_{i} \varrho_{q}+\left(\dot{f}+k^{2} f\right) \delta_{i q} .
\end{array}\right\}
$$

$f(\varrho)$ ist gleich $\frac{e^{i k \varrho}}{\varrho}$ wie in Abschnitt 2. Auf Grund des Umstandes, daß beide Paare von Feldvektoren hier als Tensoren zu behandeln sind, entsteht eine nicht unbeträchtliche Komplikation des vektoriellen Falles. Beide ,Vektoren" $V$ sind jetzt Tensoren dritten Ranges:

$$
\left.\begin{array}{l}
V_{i, p q}^{(1)}=\varepsilon_{i \alpha \beta}\left(E_{\alpha p} \bar{E}_{\beta q}+\eta H_{\alpha p} \bar{H}_{\beta q}\right), \\
V_{i, p q}^{(2)}=\varepsilon_{i \alpha \beta}\left(H_{\alpha p} \bar{E}_{\beta q}-\eta E_{\alpha p} \bar{H}_{\beta q}\right) .
\end{array}\right\}
$$

Zwischen dem ersten und den beiden letzten Indizes ist ein Komma geschrieben, um anzuzeigen, da $\beta$ nur die Divergenz, die in bezug auf den ersten Index gebildet ist, verschwindet. Beide Tensoren sind gänzlich unsymmetrisch, auch in bezug auf $p$ und $q$. Nach Benutzung von (2.8) und (4.4) werden sie:

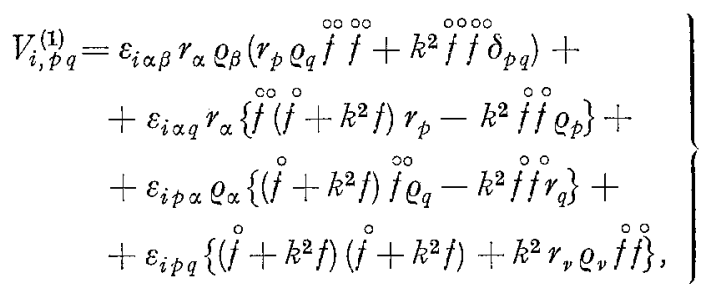

$$
\begin{aligned}
& -\frac{1}{i k} V_{i, p q}^{(2)}=\stackrel{\infty}{f} f\left(\varrho_{i} \gamma_{p} \gamma_{q}-r_{\nu} \varrho_{\nu} \gamma_{p} \delta_{i q}\right)+ \\
& +\stackrel{\circ}{f}\left(-r_{i} \varrho_{p} \varrho_{q}+r_{v} \varrho_{\nu} \varrho_{q} \delta_{i p}\right)+ \\
& +\left(\stackrel{\circ}{+}+k^{2} f\right) \stackrel{\circ}{f}\left(\varrho_{i} \delta_{p q}-\varrho_{p} \delta_{i q}\right)+ \\
& +\stackrel{\circ}{f}\left(\dot{f}+k^{2} f\right)\left(-r_{i} \delta_{p q}+r_{q} \delta_{i p}\right) .
\end{aligned}
$$

An den $f$ enthaltenden Ausdrücken ist das jeweilige Argument weggelassen worden, um die Schreibweise zu vereinfachen. Da die $V$ und ihre später abzuleitenden Tensorpotentiale $A$ alle in den $f$ und ihren Ableitungen bilinear sind, sei es stillschweigend angenommen, da $\beta$ der an erster Stelle stehende $f$-Ausdruck das Argument $r$, der zweite das 
Argument $\varrho$ hat. Trotz der Unsymmetrie erfüllen die beiden Tensoren die Beziehung

$$
V_{i, p q}^{(s)}(\vec{r}, \vec{\varrho})=-V_{i, q p}^{(s)}(\vec{\varrho}, \vec{r}) . \quad(s=1,2) .
$$

Wie das Auftreten des $\varepsilon$-Symbols zeigt, besitzt $V^{(1)}$ Pseudocharakter und könnte als Tensor vierter Stufe, mit zwei antisymmetrischen ersten Indizes, geschrieben werden. Davon ist jedoch im folgenden kein Gebrauch gemacht worden. $V^{(2)}$ ist offenbar ein gewöhnlicher Tensor dritter Stufe.

Das Ziel der folgenden Rechnungen ist nun, die Tensorpotentiale explizite aus

$$
\left.\begin{array}{rl}
V_{i, p q}^{(1)} & =\varepsilon_{i \alpha \beta} \frac{\partial}{\partial x_{\beta}} A_{\alpha, p q}^{(1)}, \\
V_{i, p q}^{(2)} & =\varepsilon_{i \alpha \beta} \frac{\partial}{\partial x_{\beta}} A_{\alpha, p q}^{(2)}
\end{array}\right\}
$$

zu ermitteln. $A^{(\mathbf{1})}$ ist jetzt ein gewöhnlicher Tensor dritter Stufe, während $A^{(2)}$ Pseudocharakter besitzt.

\section{Konstruktion des Tensorpotentials $\boldsymbol{A}_{j, p q}^{(1)}$}

Wie gerade betont, sind die beiden GIn. (4.8) von gänzlich verschiedener Form, so daß auch die Wege zur Gewinnung einzeln kurz beschrieben werden müssen. Beginnen wir mit $A^{(1)}$ und dem zugehörigen $V^{(1)}$.

Es ist klar, da $B$ man die Aufgabe, das $A$ des langen Ausdrucks (4.6) zu finden, dadurch aufteilen kann, da $B$ man solche seiner Terme, deren Divergenz separat verschwindet, auch durch einen separaten $A$-Tensor darstellen kann. Aus diesem Grunde erweist sich die folgende Aufteilung als vorteilhaft:

mit

$$
V_{i, p q}^{(1)}=U_{i, p q}+k^{2} T_{i,[p q]}
$$

$$
\begin{aligned}
& U_{i, p q}=k^{2} \varepsilon_{i \alpha \beta} \gamma_{\alpha} \gamma_{\beta} \delta_{p q} \stackrel{\circ}{\dagger}+\varepsilon_{i \alpha \beta} \gamma_{\alpha} \varrho_{\beta} \gamma_{p} \gamma_{q} \stackrel{000}{\dagger}+\varepsilon_{i p q} \stackrel{\circ}{\dagger}+ \\
& +\varepsilon_{i \alpha q} r_{\alpha} r_{p} \stackrel{00}{i}-\varepsilon_{i \alpha p} \varrho_{\alpha} \varrho_{q} i j+ \\
& +k^{2}\left\{\varepsilon_{i \alpha q} \gamma_{p}\left(\varrho_{\alpha} \stackrel{\circ}{f}+r_{\alpha} \stackrel{\infty}{f} f\right)-\varepsilon_{i \alpha p} \varrho_{q}\left(\varrho_{\alpha} f \stackrel{\infty}{f}+\gamma_{\alpha} \stackrel{\circ}{f}\right)+\right. \\
& \left.+\varepsilon_{i p q}(f f+f f)\right\}
\end{aligned}
$$

und dem, wie durch die eckigen Klammern angedeutet, in $p$ und $q$ antisymmetrischen Tensor

$$
\left.\begin{array}{rl}
T_{i,[p q]}= & {\left[\left(\varepsilon_{i \alpha p} \varrho_{q}-\varepsilon_{i \alpha q} \varrho_{p}\right) r_{\alpha}+\left(\varepsilon_{i \alpha p} \gamma_{q}-\varepsilon_{i \alpha q} r_{p}\right) \varrho_{\alpha}+\right.} \\
& \left.+\varepsilon_{i p q} \gamma_{\nu} \varrho_{v}\right] \stackrel{\circ}{\dagger}+k^{2} \varepsilon_{i p q} t f .
\end{array}\right\}
$$


Zur Erklärung dieser anfänglich recht willkürlich und umständlich erscheinenden Aufteilung sei gesagt, daß die Divergenz von $U$ verschwindet unabhängig von der Bedeutung der Funktion $f$ [definiert in (2.5)], während zum Beweis des Verschwindens der Divergenz von $T$ die Differentialgleichung (2.7) benutzt werden muß.

Es ist deshalb verständlich, daß man das Tensorpotential von $U$ direkt ablesen kann. Dieses ist:

$$
\left.\begin{array}{rl}
A_{i, p q}^{U}= & k^{2} r_{j} \delta_{p q} \stackrel{\circ}{f}+r_{i} r_{p} \varrho_{q} \stackrel{\infty}{f} f+\delta_{j p} \varrho_{q} \stackrel{\circ}{f}+ \\
& +k^{2}\left\{\varrho_{q} \delta_{j p} f \dot{\circ}-r_{p} \delta_{j q} i f\right\} .
\end{array}\right\}
$$

Der das $U$ darstellende $A$-Tensor ist natürlich nicht eindeutig, und mehrere auch die Symmetrie in $r$ und $\varrho$ erhaltende Ausdrücke lassen sich angeben.

Für das Tensorpotential $A^{T}$ von $T$ gilt nach (4.8)

$$
T_{i,[p q]}=\varepsilon_{i \alpha \beta} \frac{\partial}{\partial x_{\beta}} A_{\alpha,[p q]}^{T} \text {. }
$$

Der Tensor $T$ hat die folgenden Eigenschaften:

a) Er ist antisymmetrisch in $p, q$;

b) er bleibt bei Vertauschung von $r_{i}$ und $\varrho_{i}$ ungeändert;

c) er ist ein axialer Tensor dritter Stufe (d.h. er enthält einen $\varepsilon$-Faktor);

d) seine Divergenz bezüglich des ersten Index verschwindet (Integralibitätsbedingung).

Daraus schließt man, daß es eine Lösung $A_{i, p q}^{T}$ von (5.5) gibt mit folgenden Eigenschaften:

a) Sie ist antisymmetrisch in $p, q$;

$\beta$ ) sie bleibt bei Vertauschung von $\gamma_{i}$ und $\varrho_{i}$ ungeändert;

$\gamma$ ) sie ist ein polarer Tensor dritter Stufe (d.h. sie enthält keinen $\varepsilon$-Faktor).

Der polare Tensorcharakter von $A_{\alpha, p q}^{T}$ kann nur durch Multiplikation einer $\alpha$-, einer $p$ - und einer $q$-Komponente der Vektoren $r_{i}$ und $\varrho_{i}$ oder durch Multiplikation einer solchen Komponente mit einem KroneckerSymbol, das die beiden anderen Indizes aufnimmt, zustande kommen. Macht man einen Ansatz mit allen möglichen derartigen Kombinationen - es sind deren 14 - und verlangt die Antisymmetrie in $p$ und $q$, so tritt eine Redulktion auf den folgenden Ansatz ein

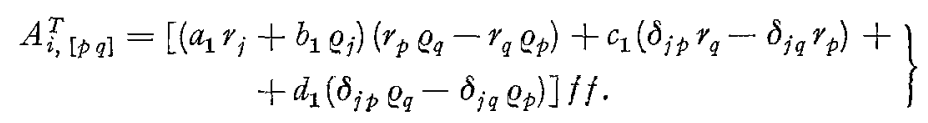


Die Koeffizienten $a_{1}, b_{1}, c_{1}, d_{1}$ hängen nur von den skalaren Größen $r, \varrho, \gamma_{\nu} \varrho_{\nu}$ (oder $r, \varrho, \Lambda$ ) und im übrigen von der Wellenzahl $k$ ab; es kann ihnen nach $\beta$ ) die Symmetrieeigenschaft

$$
a_{1}(r, \varrho, \Lambda, k)=-b_{1}(\varrho, r, \Lambda, k) ; \quad c_{1}(r, \varrho, \Lambda, k)=d_{1}(\varrho, r, \Lambda, k)
$$

auferlegt werden. Was ihre $k$-Abhängigkeit betrifft, so kommt man mit dem Rubinowiczschen Ansatz der Unabhängigkeit von $k$, der beim skalaren Problem auf (3.11) führte, nicht mehr aus. Das $V$ in (3.8) enthält nur lineare Terme in $k$, während $T_{i},[p q]$ in (5.3) quadratische Terme enthält. Die Differentiation in (5.5) schafft einen Faktor $k$ von der Differentiation von $f f$ her; einen weiteren Faktor $k$ müssen wir daher von den Koeffizienten $a_{1}, b_{1}, c_{1}, d_{1}$ her bekommen. Diese Überlegung veranlaßt uns, für diese Koeffizienten lineare Funktionen in $k$ anzusetzen. Dieser Ansatz erweist sich nun in der Tat als erfolgreich. Geht man mit ihm in (5.6) ein, setzt dann (5.6) und (5.3) in (5.5) ein und macht Koeffizientenvergleich in den Potenzen von $k$ und in den Komponenten der Vektoren $r_{i}$ und $\varrho_{i}$, so ergeben sich mehrere Gleichungen für $a_{1}, b_{1}$, $c_{1}, d_{1}$, die sich widerspruchsfrei, wenn auch etwas langwierig, lösen lassen. Es ergibt sich

$$
\begin{aligned}
& a_{1}=\frac{i k}{2 r \Lambda}-\frac{1}{2 r^{2} \Lambda}+\frac{r-\varrho}{2 r \Lambda^{2}}, \\
& b_{1}=-\frac{i k}{2 \varrho \Lambda}+\frac{1}{2 \varrho^{2} \Lambda}+\frac{r-\varrho}{2 \varrho \Lambda^{2}}, \\
& c_{1}=-\frac{i k \varrho}{\Lambda}+\frac{1}{2 \Lambda}, \\
& d_{1}=-\frac{i k r}{\Lambda}+\frac{1}{2 A} .
\end{aligned}
$$

Die Summe von (5.4) und dem mit $k^{2}$ multiplizierten Ausdruck (5.6) stellt das Tensorpotential für $V^{(\mathbf{1})}$ dar.

\section{Konstruktion des Tensorpotentials $\boldsymbol{A}_{\boldsymbol{j}, \boldsymbol{p} \boldsymbol{q}}^{(2)}$}

Wir wenden uns nun der Aufgabe zu, das zu (4.7) gehörende Tensorpotential $A^{(2)}$ herzuleiten. Auch hier ist eine Aufteilung von $V_{j, p q}^{(2)}$ möglich, und zwar setzen wir

mit

$$
-\frac{1}{i k} V_{i, p q}^{(2)}=\varepsilon_{i \alpha \beta} \frac{\partial \Phi_{\alpha p q}}{\partial x_{\beta}}+\varepsilon_{i \alpha \beta} \frac{\partial \Psi_{\alpha p q}}{\partial x_{\beta}}
$$

$$
\left.\begin{array}{rl}
\Phi_{\alpha p q}= & \frac{1}{2}\left(\varepsilon_{\alpha p m} \gamma_{q}+\varepsilon_{\alpha q m} r_{p}\right) \varrho_{m} \stackrel{\circ}{\dagger}- \\
& -\frac{1}{2}\left(\varepsilon_{\alpha p m} \varrho_{q}+\varepsilon_{\alpha q m} \varrho_{q}\right) r_{m} i f- \\
& -\frac{1}{2} k^{2} \varepsilon_{\alpha p q} f f-\frac{1}{2} r_{\nu} \varrho_{v} \varepsilon_{\alpha p q} \circ \dot{f}
\end{array}\right\}
$$


und

$$
\left.\begin{array}{rl}
\varepsilon_{i \propto \beta} \frac{\partial \Psi_{\alpha p q}}{\partial x_{\beta}}= & \frac{\mathbf{1}}{2}\left(r_{p} \varrho_{q}+r_{q} \varrho_{p}\right)\left(r_{i} \dot{f} f-\varrho_{i} \dot{f} \dot{f}\right)- \\
& +\frac{1}{2}\left[\delta_{i p}\left(\varrho_{q}-r_{q}\right)+\delta_{i q}\left(\varrho_{p}-r_{p}\right)\right] \stackrel{\circ}{f}- \\
& +k^{2}\left(\varrho_{i} f \dot{\circ}-r_{i} \dot{f} f\right) \delta_{p q} .
\end{array}\right\}
$$

(6.3) stellt eine Differentialgleichung für $\Psi_{\alpha, p q}$ dar. Ihre rechte Seite hat folgende vier Eigenschaften:

a) Sie ist symmetrisch in $p$ und $q$;

b) sie ändert bei Vertauschung von $\gamma_{i}$ und $\varrho_{i}$ ihr Vorzeichen;

c) sie ist ein polarer Tensor dritter Stufe;

d) ihre Divergenz verschwindet (Integrabilitätsbedingung).

Es gibt daher eine Lösung von (6.3) mit den Eigenschaften:

a) Sie ist symmetrisch in $p$ und $q$;

$\beta)$ sie ändert bei Vertauschung von $r_{i}$ und $\varrho_{i}$ ihr Vorzeichen;

$\gamma)$ sie ist ein axialer Tensor und enthält somit einen $\varepsilon$-Faktor.

Dies legt folgenden Ansatz nahe

$$
\begin{aligned}
\Psi_{\alpha p q}= & \varepsilon_{\alpha m n} \gamma_{m} \varrho_{n}\left(r_{p} \varrho_{q}+r_{q} \varrho_{p}\right) f f a_{2}+ \\
& +\varepsilon_{\alpha m n} \gamma_{m} \varrho_{n} \gamma_{p} \gamma_{q} f f b_{2}+ \\
& +\varepsilon_{\alpha m n} r_{m} \varrho_{n} \varrho_{p} \varrho_{q} f f c_{2}+ \\
& +\left[\varepsilon_{\alpha m p} r_{m}\left(\varrho_{q}-r_{q}\right)+\varepsilon_{\alpha m q} r_{m}\left(\varrho_{p}-r_{p}\right)\right] f f d_{2}+ \\
& +\left[\varepsilon_{\alpha m p} \varrho_{m}\left(r_{q}-\varrho_{q}\right)+\varepsilon_{\alpha m q} \varrho_{m}\left(r_{p}-\varrho_{p}\right)\right] f f e_{2}+ \\
& +\varepsilon_{\alpha m n} r_{m} \varrho_{n} \delta_{p q} f f f_{2}
\end{aligned}
$$

mit

$$
\left.\begin{array}{ll}
a_{2}(r, \varrho)=a_{2}(\varrho, r), & b_{2}(r, \varrho)=c_{2}(\varrho, r), \\
d_{2}(r, \varrho)=-e_{2}(\varrho, r), & f_{2}(r, \varrho)=f_{2}(\varrho, r) .
\end{array}\right\}
$$

Die sechs Koeffizienten $a_{2}$ bis $f_{2}$ hängen im übrigen nur von $r, \varrho, r_{\nu} \varrho_{v}$ bzw. $\mathcal{A}$ und von $k$ ab. Führt man die Differentiationen von $f(r)$ und $f(\varrho)$ in (6.3) aus, so wird die rechte Seite ein Polynom in $i k$ vom Grad 3, multipliziert mit $f f$. Wir erwarten daher mit analoger Begründung wie im letzten Abschnitt, daß die Koeffizienten $a_{2}$ bis $f_{2}$ als Polynome zweiten Grades in $i k$ dargestellt werden können. 
Einsetzen von (6.4) in (6.3) gibt mit grundsätzlich einfacher, aber langwieriger Rechnung das Resultat

$$
\begin{aligned}
a_{2}= & \frac{(i k)^{2}}{2 r \varrho \Lambda}-\frac{i k(r+\varrho)}{2 r \varrho \Lambda}\left[\frac{1}{r \varrho}+\frac{1}{2 \Lambda}\right]+ \\
& +\frac{1}{2 r^{2} \varrho^{2} \Lambda}-\frac{(r-\varrho)^{2}}{4 r^{2} \varrho^{2} \Lambda^{2}}-\frac{(r-\varrho)^{2}}{2 r \varrho \Lambda^{3}}, \\
b_{2}= & -\frac{i k}{2 r \Lambda^{2}}+\frac{1}{2 r^{2} \Lambda^{2}}+\frac{\varrho-r}{r \Lambda^{3}}, \\
c_{2}= & -\frac{i k}{2 \varrho \Lambda^{2}}+\frac{1}{2 \varrho^{2} \Lambda^{2}}+\frac{r-\varrho}{\varrho \Lambda^{3}} \\
d_{2}= & -\frac{i k}{2 r \Lambda}+\frac{1}{4 r^{2} \Lambda}+\frac{\varrho-2 r}{4 r \Lambda^{2}}, \\
e_{2}= & \frac{i k}{4 \varrho \Lambda}-\frac{1}{4 \varrho^{2} \Lambda}+\frac{2 \varrho-r}{4 \varrho \Lambda^{2}}, \\
f_{2}= & \frac{k^{2}}{\Lambda} .
\end{aligned}
$$

\section{Herleitung der Larmor-Tedoneschen und verwandter Formeln. (Das räumliche Residuum)}

Sei $E_{i}(r), H_{i}(r)$ ein elektromagnetisches Feld, welches im Halbraum $\mathrm{H}_{2}$ regulär ist und dort der Ausstrahlungsbedingung genügt:

$$
\left.\begin{array}{l}
r E_{i}, r H_{i} \text { beschränkt für } r \rightarrow \infty \text { in } H_{2}, \\
r E_{i}+\varepsilon_{i k l} r_{k} H_{l} \rightarrow 0 \\
r H_{i}-\varepsilon_{i k l} \gamma_{k} E_{l} \rightarrow 0
\end{array}\right\} \text { für } r \rightarrow \infty \text { in } H_{2} .
$$

Die Lösung (7.2) der adjungierten Maxwellschen Gln. (7.1) genügt der adjungierten Ausstrahlungsbedingung, d.h. (7.1) und (7.2), wenn man dort $r$ durch $\varrho, E_{i}$ durch $\bar{E}_{i q}$ und $H_{i}$ durch $-\eta \bar{H}_{i q}$ ersetzt.

Das bilineare Feld

$$
V_{i q}=\varepsilon_{i \alpha \beta}\left[H_{\alpha} \bar{E}_{\beta q}-\eta E_{\alpha} \bar{H}_{\beta q}\right]
$$

hat wegen der angenommenen Ausstrahlungsbedingung die Eigenschaft $r \varrho V_{i q} \rightarrow 0$ für $r \approx \varrho \rightarrow \infty$, und daher verschwindet das Flächenintegral $\int V_{i q} d F_{i}$, wenn man es über irgendeinen Teil der „unendlich fernen Kugel" $H_{2}$ erstreckt.

Wir schließen nun eine kleine Kugel $K$ mit dem Mittelpunkt $P$ vom Halbraum $H_{2}$ aus; im verbleibenden Raumgebiet $H_{2}-K$ ist $V_{i p}$ regulär und $\frac{\partial}{\partial x} V_{i p}=0$. Das Integral dieses Ausdrucks über $H_{2}-K$ verschwindet daher ebenfalls. Anwendung des Gaußschen Satzes gibt somit wegen des Verhaltens von $V_{i p}$ im Unendlichen

$$
\int_{\sigma+\bar{\sigma}} V_{i q} d F_{i}=\oint_{K} V_{i q} d F_{i} \text {. }
$$


Das Flächenelement $d F_{i}$ des linken Integrals sei so gerichtet, wie in Fig .1 angedeutet; das Flächenintegral rechts erstreckt sich über die Oberfläche der Kugel $K$ mit nach außen gerichteter Normalen.

Das Integral rechter Hand läßt sich im Limes $K \rightarrow P$ leicht berechnen. Zunächst ist nach (7.3) und (4.4)

$$
\begin{aligned}
\oint_{K} V_{i} d F_{i}= & \oint_{K} \varepsilon_{i \alpha \beta}\left[H_{\alpha}\left(\stackrel{\infty}{f} \varrho_{\beta} \varrho_{q}+\left(\stackrel{\circ}{f}+k^{2} f\right) \delta_{\beta q}\right)+\right. \\
& \left.+i k E_{\alpha} \varepsilon_{\beta q \gamma} \varrho_{\gamma} \stackrel{\circ}{f}\right] d F_{i} .
\end{aligned}
$$

Setzt man $d F_{i}=\varrho_{i} d F / \varrho$, so erkennt man, daß der Term mit $\stackrel{\circ}{f}$ keinen Beitrag liefert, da $\varepsilon_{i \alpha \beta} \varrho_{\beta} \varrho_{i}=0$. Der Term mit $k^{2}$ gibt einen Beitrag der Ordnung $Q$, der im Limes $K \rightarrow 0$ verschwindet. Der Faktor von $E_{\alpha}$ ist von der Ordnung $\varrho^{-2} d F$. Daher darf $E_{\alpha}(Q)$ durch $E_{\alpha}(P)$ ersetzt werden mit einem Fehler, der wieder in der Grenze verschwindet. Der Faktor von $H_{\alpha}$ ist jedoch von der Ordnung $\varrho^{-\mathbf{3}} d F_{i}$ und deshalb ist es nötig, $H_{\alpha}$ bis zu linearen Gliedern zu entwickeln, $H_{\alpha}(Q)=H_{\alpha}(P)+$ $\varrho_{i} \times \frac{\partial H_{\alpha}(P)}{\partial x_{j}}+\cdots ; H_{\alpha}(P)$ gibt wieder keinen Beitrag, da $\oint d F_{i}=0$. Somit bleibt

Nun ist

$$
\begin{aligned}
& \oint_{K} V_{i q} d F_{i}=\varepsilon_{i \alpha \beta} \frac{\partial H_{\alpha}(P)}{\partial x_{j}} \stackrel{\circ}{j} \delta_{q q} \oint \varrho_{j} \varrho_{i} \frac{d F}{\varrho}+ \\
& +i k \varepsilon_{i \alpha \beta} E_{\alpha}(P) \varepsilon_{\beta q \gamma} \stackrel{\circ}{\oint} \varrho_{\gamma} \varrho_{i} \frac{d F}{\varrho}+0(\varrho) \text {. }
\end{aligned}
$$

$$
\oint \varrho_{i} \varrho_{j} d F=\frac{4 \pi}{3} \varrho^{4} \delta_{i j} \quad \text { and } \quad \lim _{\varrho \rightarrow 0} \varrho^{3} f(\varrho)=-1
$$

und somit nach einigen Vereinfachungen wegen (2.2)

$$
\oint V_{i q} d F=-\frac{4 \pi}{3} \varepsilon_{i \alpha q} \frac{\partial H_{\alpha}(P)}{\partial x_{i}}+\frac{8 \pi}{3} i k E_{q}(P)=4 \pi i k E_{q}(P) .
$$

Zusammenfassend gilt also

$$
4 \pi i k E_{q}(P)=\int_{\sigma \neq \bar{\sigma}} \varepsilon_{i \alpha \beta}\left[H_{\alpha} \bar{E}_{\beta q}-\eta E_{\alpha} \bar{H}_{\beta q}\right] d F_{i} .
$$

Entsprechend erhält man, wenn $\bar{E}_{\beta q}^{\prime}$ und $\bar{H}_{\beta q}^{\prime}$ das Feld eines Dipols im Punkte $P^{\prime}$ im Halbraum $H_{1}$ ist,

$$
0=\int_{\sigma+\bar{\sigma}} \varepsilon_{i \alpha \beta}\left[H_{\alpha} \bar{E}_{\beta q}^{\prime}-\eta E_{\alpha} \bar{H}_{\beta q}^{\prime}\right] d F_{i},
$$

da dann im Halbraum $H_{2}$ keine Singularität von $V_{i}$ liegt. Daher gilt, mit beliebigern $\zeta$,

$$
4 \pi i k E_{q}(P)=\int_{\sigma+\bar{\sigma}} \varepsilon_{i \alpha \beta}\left[H_{\alpha}\left(\bar{E}_{\beta q}+\zeta \bar{E}_{\beta q}^{\prime}\right)-\eta E_{\alpha}\left(\bar{H}_{\beta q}+\zeta \bar{H}_{\beta q}^{\prime}\right)\right] d F_{i}
$$

oder

$$
4 \pi i k E_{q}(P)=\int_{\sigma+\bar{\sigma}}\left(V_{i_{q}}^{(2)}+\zeta V_{i q}^{(2)^{\prime}}\right) d F_{i}
$$


Aus der Symmetrie der Maxwellschen Gleichungen oder durch Bi1dung der Rotation des letzten Ergebnisses erhält man

$4 \pi i k H_{q}(P)=\int_{\sigma+\bar{\sigma}} \varepsilon_{i \alpha \beta}\left[-\eta H_{\alpha}\left(\bar{H}_{\beta q}+\zeta \bar{H}_{\beta q}^{\prime}\right)-E_{\alpha}\left(\bar{E}_{\beta q}+\zeta \bar{E}_{\beta q}^{\prime}\right)\right] d F_{i}$

oder

$$
-4 \pi i k H_{q}(P)=\int_{\sigma+\bar{\sigma}}\left(V_{i q}^{(1)}+\zeta V_{i q}^{(1) \prime}\right) d F_{i},
$$

wenn $V_{i q}^{(1)}$ analog zu $V_{i q}^{(2)}$ in (7.3), d.h. nach (4.5) mit willkürlichem Feld $E_{\alpha}, H_{\alpha}$ an Stelle des Dipolfeldes $E_{\alpha p}, H_{\alpha p}$ definiert ist.

Das Feld $E_{q}(P), H_{q}(P)$ ist somit durch die Tangentialkomponenten dieses Feldes auf $\sigma+\bar{\sigma}$ ausgedrückt. Eine Vereinfachung ergibt sich für ebene Schirme $\sigma$, wenn zugleich $\bar{\sigma}$ als eben angenommen wird. Man wählt für $\bar{E}_{p q}^{\prime}, \bar{H}_{p q}^{\prime}$ das Feld eines Dipols im Spiegelpunkt $P^{\prime}$ von $P$ und $\zeta=+1$ oder -1 . Dann folgen die Formelpaare

$$
\begin{aligned}
& 2 \pi i k E_{q}(P)=\int_{\sigma+\bar{\sigma}} \varepsilon_{i \alpha \beta} H_{\alpha} \bar{E}_{\beta q} d F_{i}, \\
& 2 \pi i k H_{q}(P)=-\eta \int_{\sigma+\bar{o}} \varepsilon_{i \alpha \beta} H_{\alpha} \bar{H}_{\beta q} d F_{i}, \\
& 2 \pi i k E_{q}(P)=-\eta \int_{\sigma+\bar{\sigma}} \varepsilon_{i \alpha \beta} E_{\alpha} \bar{H}_{\beta q} d F_{i}, \\
& 2 \pi i k H_{q}(P)=-\int_{\sigma+\bar{\sigma}} \varepsilon_{i \alpha \beta} E_{\alpha} \bar{E}_{\beta q} d F_{i} .
\end{aligned}
$$

Mit diesen Formeln läßt sich also das elektromagnetische Feld $E_{q}(P)$, $H_{q}(P)$ im Halbraum $H_{2}$ durch die Tangentialkomponenten entweder von $E_{\alpha}(Q)$ allein oder von $H_{\alpha}(Q)$ allein auf $\sigma+\bar{\sigma}$ ausdrücken. (7.14) und (7.15) folgen auseinander auch über die Maxwellschen Gln. (2.2). Dasselbe gilt für (7.16) und (7.17).

Die Gln. (7.14) bis (7.16) finden sich bei LUNeBerg und in SOMMERFELDs Optik ${ }^{7,8}$. In dem letzteren Werk sind zwar nur (7.15) und (7.16) explizit angegeben; die anderen beiden Formeln folgen aber dann, wie eben erwähnt, in trivialer Weise aus den Maxwellschen Gleichungen.

\section{Der Beitrag des direkten Lichtes (Das zweidimensionale Residuum)}

Die Beugung einer von $O$ im Halbraum $H_{1}$ ausgehenden elektrischen. Dipolwelle läßt sich näherungsweise - in einer der Kirchhoffschen entsprechenden Näherung - behandeln, wenn man in (7.10) und (7.12).

7 Luneberg, R. K.: Mathematical Theory of Optics, 1944 (Brown University).

8 Sommerfeld, A.: Vorlesungen über theoretische Physik, Bd. IV: Optik. Wiesbaden 1950. 
unter dem Integral $E_{\alpha p}$ und $H_{\alpha p}$ statt $E_{\alpha}$ und $H_{\alpha}$ schreibt und nur über die Öffnung $\bar{\sigma}$ integriert. Man nimmt also an der unteren Schirmfläche verschwindendes Feld an und setzt das Feld in der Öffnung gleich dem der einfallenden Welle. Dann ergibt sich wegen (4.5) für das Feld im Halbraum $\mathrm{H}_{2}$

$$
\begin{aligned}
& 4 \pi i k E_{q p}^{(2)}(P)=\int_{\bar{\sigma}}\left[V_{i, p q}^{(2)}+\zeta V_{i, p q}^{(2)}\right] d F_{i}, \\
& 4 \pi i k H_{q p}^{(1)}(P)=\int_{\sigma}\left[V_{i, p q}^{(1)}+\zeta V_{i, p q}^{(1)^{\prime}}\right] d F_{i} .
\end{aligned}
$$

Es ist ausdrücklich zu bemerken, daß (7.10) und (7.12) dasselbe elektromagnetische Feld darstellen, d.h., daß $E_{q}$ aus (7.10) und $H_{q}$ aus (7.12) die Maxwellschen Gleichungen erfüllen. Dies ist für $E_{q p}^{(2)}$ aus (8.1) und $H_{q p}^{(1)}$ aus (8.2) nicht mehr der Fall. Sie stellen vielmehr zwei verschiedene Näherungen dar. Das zu Näherung (8.2) gehörende elektrische Feld $E_{q p}^{\langle 1\rangle}$ berechnet sich aus $H_{q p}^{(1)}(P)$ und der zweiten Maxwellschen Gleichung in (2.2); analog berechnet sich das zur Näherung (8.1) gehörende magnetische Feld $H_{q p}^{(2)}$.

Es sei zunächst angenommen, daß die Linie, welche den Lichtpunkt $O$ mit dem Aufpunkt $P$ verbindet, die beugende Öffnung nicht durchsetzt. Der Aufpunkt $P$ liege also im geometrischen Schatten. Dann ist für alle Öfnungspunkte $Q$ der Ausdruck $A=r \varrho+r_{v} \varrho_{v}$ von Null verschieden, die Vektorpotentiale $A$ sind für den ganzen Integrationsbereich regulär und man kann daher nach Einsetzen der Vektorpotentiale in (8.1) und (8.2) den Stokesschen Satz anwenden. Es ergibt sich so

$$
\begin{aligned}
4 \pi i k E_{q p}^{(2)}(P) & =-\oint\left[A_{j, p q}^{(2)}+\zeta A_{j, p q}^{(2)}\right] d x_{j}, \\
4 \pi i k H_{q p}^{(1)}(P) & =-\oint\left[A_{j, p q}^{(1)}+\zeta A_{j, p q}^{(1)}\right] d x_{j},
\end{aligned}
$$

wobei nun die Integration entlang des Öffnungsrandes zu erstrecken ist.

Wenn bei allmählicher Verschiebung der Aufpunkt $P$ aus dem Schatten tritt und von dem direkten Licht erreicht wird, tritt in allen $A$-Tensoren (aber nicht in den $A^{\prime}$-Tensoren) eine Singularität auf, da der Nenner $A=r \varrho+\gamma_{\nu} \varrho_{v}$ auf der Verbindungslinie von $O$ nach $P$ verschwindet. Um auch jetzt den Stokesschen Satz anwenden zu können, muß man erst von der beugenden Öffnung eine kleine Kreisscheibe ausnehmen. Sie soll senkrecht zum direkten Strahl $O P$ sein und von ihm im Mittelpunkt durchsetzt werden. Das Integral (8.1) oder (8.2) über diese Kreisscheibe geht gegen Null, wenn der Radius $\xi$ der Kreisscheibe gegen Null geht. Der Stokessche Satz wird nun auf die Öffnung mit Ausschluß dieser Kreisscheibe angewandt. Dann erhält man wieder die Integrale (8.3), (8.4) längs des Öffnungsrandes, zusätzlich aber Integrale im entgegengesetzten Umlaufsinn entlang des Kreisscheibenrandes. 
Für $\xi \rightarrow 0$ geben nur solche Terme in $A_{j, p q}^{(1)}$ und $A_{j, p q}^{(2)}$ einen nicht verschwindenden Beitrag, die einen Nenner $A$ oder $A^{2}$ oder $\Lambda^{3}$ enthalten. $Z u$ dem nach (8.3) oder (8.4) berechneten Feld tritt also noch ein Zusatzbeitrag

$$
\begin{aligned}
& 4 \pi i k E_{q p}^{(2 d)}(P)=\lim _{\xi \rightarrow 0} \oint A_{j, p q}^{(2)} d \xi_{j}, \\
& 4 \pi i k H_{q p}^{(1 d)}(P)=\lim _{\xi \rightarrow 0} \oint A_{j, q p}^{(\mathbf{l})} d \xi_{j}
\end{aligned}
$$

mit Integration längs der Kreisscheibe. Der Index $d$ soll andeuten, da $B$ diese Integrale das direkte Licht geben.

Wir behandeln nun ausführlicher den Fall (8.6). Die geometrischen Verhältnisse sind durch Fig. 2 erläutert. Sei

$$
\vec{r}=\overrightarrow{r_{0}}+\vec{\xi}, \quad \vec{e}=\vec{\varrho}_{0}+\vec{\xi}
$$

und $\vec{r}_{0}, \vec{\varrho}_{0}$ entgegengesetzt gerichtet, $\vec{\xi}$ senkrecht

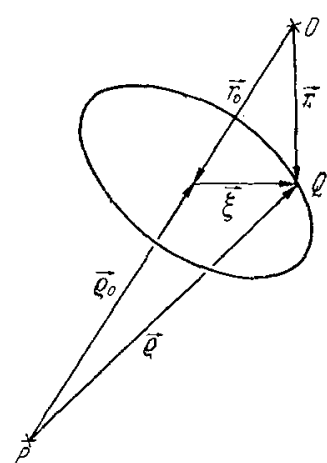

Fig. 2. Zur Berechnung des direkten Lichtes $\mathrm{zu} \vec{r}_{0}$ und $\overrightarrow{\varrho_{0}}$ vom konstanten Betrag $\xi$. Setzt $\operatorname{man} A_{j, p q}^{(1)}$ in (8.6) ein, so bleiben im Limes $\xi \rightarrow 0$ nur die Terme, die von $k^{2} A_{j,[p q]}^{T}[$ s. (5.6)] herrühren. Man erhält daher

$$
\left.\begin{array}{rl}
4 \pi i k H_{q p}^{(1 a)}(P)= & \lim _{\xi \rightarrow 0} k^{2} f f \oint\left\lceil\left(a_{1} r_{j}+b_{1} \varrho_{j}\right)\left(r_{p} \varrho_{q}-r_{q} \varrho_{p}\right)\right. \\
& \left.+c_{1}\left(\delta_{j p} \varrho_{q}-\delta_{j q} r_{p}\right)+d_{1}\left(\delta_{j p} \varrho_{q}-\delta_{j q} \varrho_{p}\right)\right] d \xi_{j} .
\end{array}\right\}
$$

Um die Integrationen auszuführen, setzt man (8.7) in (8.8) ein. Es treten dann Integrale der Gestalt

$$
I_{k}=\oint d \xi_{k}, \quad I_{i k}=\oint \xi_{i} d \xi_{k}, \quad I_{i j k}=\oint \xi_{i} \xi_{j} d \xi_{k},
$$

auf. Man berechnet sie am einfachsten, indem man sie durch Anwendung des Stokesschen Satzes wieder auf Flächenintegrale zurückführt. Bezeichnet man mit $\vec{R}$ den Vektor von $O$ nach $P$, so ist $d F_{l}=-\frac{R_{l}}{R} d F$. Also wird für ein skalares Feld $B$

$$
\oint B d \xi_{k}=\int \varepsilon_{k l m} \frac{\partial B}{\partial \xi_{m}} d F_{l}=\frac{R_{l}}{R} \int \varepsilon_{k m l} \frac{\partial B}{\partial \xi_{m}} d F .
$$

Anwendung dieser Formel liefert unmittelbar

$$
I_{k}=0, \quad I_{i k}=\varepsilon_{k i l} \frac{R_{l}}{R} \cdot \xi^{2} \pi, \quad I_{i j k}=0 .
$$

Damit folgt aus (8.8)

$$
4 \pi i k H_{q p}^{(1 d)}(P)=2 \lim _{\xi \rightarrow 0} k^{2} f f\left(c_{1}+d_{1}\right) \xi^{2} \pi \varepsilon_{p q l} \frac{R_{l}}{R} .
$$


Nun ist nach (5.8)

und für kleine $\xi$

$$
c_{1}+d_{1}=[-i k(r+\varrho)+1] \cdot \frac{1}{\Lambda}
$$

Daher folgt aus (8.12)

$$
\Lambda \approx \frac{R^{2}}{2 v_{0} \varrho_{0}} \xi^{2}
$$

$$
4 \pi i k H_{q p}^{(1 d)}(P)=-4 \pi k^{2} \stackrel{\circ}{f}(R) \varepsilon_{p q l} R_{l} .
$$

Wie ein Vergleich mit (2.8) zeigt, ist $H_{q p}^{(1 d a)}(P)$ gerade das Magnetfeld im Punkte $P$ für einen in $O$ befindlichen schwingenden elektrischen Dipol. Das zweidimensionale Residuum des Vektorpotentials $A_{j, p q}^{(1)}$ gibt also genau das direkte Licht (durch den Index $d$ in $H_{q p}^{(1)}$ angedeutet).

Dieses Ergebnis ist nicht weiter überraschend, da bei Ausweitung des Öffnungsrandes ins Unendliche ja genau das direkte Licht herauskommen muß. Dann gehen aber die Integrale (8.3) und (8.4) gegen Null, und das von der Öffnungsgröße unabhängige Residuum des Zusatzintegrals bleibt übrig; es muß also gerade das direkte Licht geben, auch bei endlicher Öffnung. Auf jeden Fall gibt aber das Resultat (8.15) eine Kontrolle für den berechneten Ausdruck $A_{j, p q}^{(1)}$.

Etwas langwieriger ist die entsprechende Rechnung für das kompliziertere Vektorpotential $A_{i, p q}^{(2)}$. Sie führt ebenfalls auf das direkte Licht und gibt wieder eine wirksame Kontrolle der in (6.6) angegebenen Koeffizienten.

\section{Schlußfolgerungen und Bemerkungen}

Der Umstand, daß beim vektoriellen Problem Tensoren dritter Stufe $V_{i, p q}^{(s)}$ an die Stelle des Vektors (3.8) beim skalaren Problem treten, läßt natürlich auch kompliziertere Vektorpotentiale erwarten. Tatsächlich sind auch die Vektorpotentiale (5.4), (5.6) mit (5.8) und (6.2), (6.4) mit (6.6) weit weniger einfach als (3.8).

Immerhin ist eine nicht unbeträchtliche Vereinfachung möglich, wenn man berücksichtigt, daß die Kirchhoffsche Näherung nur sinnvoll bleibt, solange sowohl die Öffnungsdurchmesser als auch die Abstände $r$ und $\varrho$ des Lichtpunktes und des Aufpunkts vom Öffnungsrand groß gegen die Wellenlänge sind. Man bleibt daher in dieser Näherung, wenn man in den Potentialen $k r \gg 1$ und $k \varrho \gg 1$ voraussetzt und damit alle Glieder in $\operatorname{den} A_{\alpha, p q}^{(s)}(S=1,2)$, die nicht den Faktor $k^{3} f f$ enthalten, streicht. Dann entsteht

$$
\left.\begin{array}{rl}
A_{\alpha, p q}^{(1)}= & i k^{3} f f\left[\bar{r}_{\alpha} \delta_{p q}-\bar{r}_{\alpha} \bar{r}_{p} \bar{\varrho}_{q}+\delta_{\alpha p} \bar{\varrho}_{q}-\delta_{\alpha q} \bar{r}_{p}+\right. \\
& +\frac{1}{2 N}\left(\bar{r}_{\alpha}-\bar{\varrho}_{\alpha}\right)\left(\bar{r}_{p} \bar{\varrho}_{q}-\bar{r}_{q} \bar{\varrho}_{p}\right)+ \\
& \left.+\frac{1}{N}\left(\delta_{\alpha q}\left(\bar{r}_{p}+\bar{\varrho}_{p}\right)-\delta_{\alpha p}\left(\bar{r}_{q}+\bar{\varrho}_{q}\right)\right)\right] ;
\end{array}\right\}
$$




$$
\left.\begin{array}{rl}
A_{\alpha, p q}^{(2)}= & i \hbar^{3} f f\left[\frac{1}{2}\left(\varepsilon_{\alpha p m} \bar{r}_{q}+\varepsilon_{\alpha q m} \bar{r}_{p}\right) \bar{\varrho}_{m}-\right. \\
& -\frac{1}{2}\left(\varepsilon_{\alpha p m} \bar{\varrho}_{q}+\varepsilon_{\alpha q m} \bar{\varrho}_{p}\right) \bar{r}_{m}+ \\
& +\frac{1}{2} \varepsilon_{\alpha p q}\left(1-\bar{r}_{\nu} \bar{\varrho}_{\nu}\right)-\varepsilon_{\alpha m n} \bar{r}_{m} \bar{\varrho}_{n} \frac{\delta p q}{N}+ \\
& \left.+\varepsilon_{\alpha m n} \bar{r}_{m} \bar{\varrho}_{n} \frac{1}{2 N}\left(\bar{r}_{p} \bar{\varrho}_{q}+\bar{r}_{q} \bar{\varrho}_{p}-2 \delta_{p q}\right)\right] .
\end{array}\right\}
$$

$N$ ist eine Abkürzung für die dimensionslose Größe $N=\Lambda / r \varrho=1+\bar{\gamma}_{\nu} \bar{\varrho}_{\gamma}$. Die überstrichenen Komponenten $\bar{\gamma}_{i}, \bar{\varrho}_{i}$ sind Abkürzungen für $r_{i} / r, \varrho_{i} / \varrho$, also Komponenten der Einheitsvektoren in Richtung der Vektoren $r_{i}, \varrho_{i}$.

Bemerkenswert an den Darstellungen (9.1) und (9.2) der Vektorpotentiale ist, daß die Abstände $r, \varrho$ nur in den Faktoren $f(r) f(\varrho)$ eingehen. Der Inhalt der eckigen Klammern hängt nur von den Richtungen der Verbindungen vom Randpunkt der Öffnung zu Lichtquelle und Aufpunkt ab. Damit bestätigt sich auch hier die Youngsche Deutungsmöglichkeit der Beugungserscheinungen einerseits, ihre Äquivalenz mit der Kirchhoffschen Näherung, also im wesentlichen dem Huygenschen Prinzip, andererseits. Und schließlich wird präzisiert, in welcher Weise ein Element des Öffnungsrandes die in der Richtung $\bar{r}_{i}$ einfallende Welle nach der Richtung $\bar{\varrho}_{i}$ ",reflektiert". Dieser Reflexionsfaktor ist durch den Ausdruck $A_{\alpha, p q}^{(s)} d x_{\alpha} / f f$ gegeben, wenn die $d x_{\alpha}$ die Komponenten des vektoriellen Linienelements sind; er hängt, wie schon erwähnt, nur von $\bar{\gamma}_{i}, \bar{\varrho}_{i}$ und $d x_{i}$ ab.

Ist speziell die einfallende Welle eine ebene Welle, und liegt der Aufpunkt $P$ im Unendlichen, so sind die Inhalte der eckigen Klammern in (9.1) und (9.2) längs des Öffnungsrandes konstant. Es kommt also für die Berechnung des gebeugten Feldes im wesentlichen auf das Integral von $f f d x_{\alpha}$ längs des Öffnungsrandes an.

Zum Schluß mögen noch einige mathematische Gedanken hier erwähnt werden. Aus dem Verschwinden der Divergenz des $V$-Vektors folgt im Prinzip natürlich die Existenz eines Vektorpotentials sofort, und zwar sowohl im skalaren wie im vektoriellen Beugungsproblem, gleichgültig was die Diemensionszahl des Raumes ist, in dem das Problem formuliert ist. Aber daß diese Potentiale nicht nur im Prinzip, sondern in manchen Fällen tatsächlich auch ganz einfach explizite ermittelt werden können, ist eigentlich ein unerwarteter Umstand. Die Methode ist dabei die Verallgemeinerung der Rubinowiczschen Methode, derzufolge alle Potentiale als Polynome, d.h. als abbrechende Reihen in $k$ bestimmt werden. Der Ansatz für irgendeine Koeffizientenfunktion eines Tensorpotentials:

$$
a=\left(a^{(s)} k^{s}+a^{(s-1)} k^{s-1}+\cdots+a^{(0)}\right) f(y) f(\varrho),
$$


wo $s$ um 1 niedriger ist als die höchste in $V$ vorkommende Potenz von $k$, führt zum Ziel im Falle der skalaren und der vektoriellen Beugung im dreidimensionalen Raume, und wie wir uns überzeugt haben, auch in allen Räumen ungerader Dimensionszahl.

In Räumen gerader Dimensionszahl, also besonders in dem interessanten zweidimensionalen Fall, versagt diese Methode gänzlich, und es gelingt nicht mehr, das Vektorpotential explizite als ein Polynom in $k$ darzustellen. Der unmittelbare mathematische Grund dafür ist, daß im gerade-dimensionalen Falle die $f(r)$ Hankelsche Funktionen mit ganzzahligem Index werden, d.h. also Funktionen, die einer Differentialgleichung erster Ordnung nicht genügen. Zwar kann man im zweidimensionalen Falle das pseudoskalare Potential $A_{12}$ mit Hilfe seiner Poisson-Gleichung als Integral in geschlossener Form darstellen, aber der Vorteil, die Anzahl der Integrationen des Kirchhoff-Integrals um eine verringert $\mathrm{zu}$ haben, geht hier natürlich verloren. Es ist anzunehmen, daß das Versagen des Young-Rubinowiczschen Gedankens in geraden Dimensionen mit den bekannten Schwierigkeiten des Huygensschen Prinzips in eben diesen Räumen zusammenhängt. 